\title{
A simulation study to compare different estimation approaches for network meta-analysis and corresponding methods to evaluate the consistency assumption
}

\author{
Corinna Kiefer ${ }^{1}$, Sibylle Sturtz ${ }^{1}$ and Ralf Bender $1,2^{*}$
}

\begin{abstract}
Background: Network meta-analysis (NMA) is becoming increasingly popular in systematic reviews and health technology assessments. However, there is still ambiguity concerning the properties of the estimation approaches as well as for the methods to evaluate the consistency assumption.

Methods: We conducted a simulation study for networks with up to 5 interventions. We investigated the properties of different methods and give recommendations for practical application. We evaluated the performance of 3 different models for complex networks as well as corresponding global methods to evaluate the consistency assumption. The models are the frequentist graph-theoretical approach netmeta, the Bayesian mixed treatment comparisons (MTC) consistency model, and the MTC consistency model with stepwise removal of studies contributing to inconsistency identified in a leverage plot.

Results: We found that with a high degree of inconsistency none of the evaluated effect estimators produced reliable results, whereas with moderate or no inconsistency the estimator from the MTC consistency model and the netmeta estimator showed acceptable properties. We also saw a dependency on the amount of heterogeneity. Concerning the evaluated methods to evaluate the consistency assumption, none was shown to be suitable.

Conclusions: Based on our results we recommend a pragmatic approach for practical application in NMA. The estimator from the netmet a approach or the estimator from the Bayesian MTC consistency model should be preferred. Since none of the methods to evaluate the consistency assumption showed satisfactory results, users should have a strong focus on the similarity as well as the homogeneity assumption.
\end{abstract}

Keywords: Consistency assumption, Indirect comparison, Mixed treatment comparison, Multiple treatments meta-analysis, Network meta-analysis, Simulation study

\section{Background}

The combination of the results of several studies comparing the same two interventions is known as meta-analysis. The concept of meta-analysis and the corresponding methods are well established in medical statistics. However, in the recent years new methods for indirect comparisons have become more and more popular $[1,2]$.

${ }^{*}$ Correspondence: Ralf.Bender@iqwig.de

'Institute for Quality and Efficiency in Health Care (IQWiG), Im Mediapark 8,

D-50670 Cologne, Germany

2Faculty of Medicine, University of Cologne, Joseph-Stelzmann-Str. 20,

D-50931 Cologne, Germany

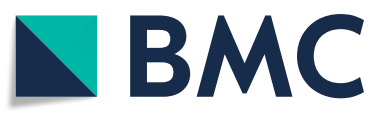
(c) The Author(s). 2020 Open Access This article is distributed under the terms of the Creative Commons Attribution 4.0
International License (http://creativecommons.org/licenses/by/4.0/), which permits unrestricted use, distribution, and reproduction in any medium, provided you give appropriate credit to the original author(s) and the source, provide a link to the Creative Commons license, and indicate if changes were made. The Creative Commons Public Domain Dedication waiver (http://creativecommons.org/publicdomain/zero/1.0/) applies to the data made available in this article, unless otherwise stated.
These comprise both the adjusted indirect comparison of two interventions, which have not been compared directly in a head-to-head-trial, and the simultaneous comparison of more than two interventions in a network of interventions.

For all indirect comparisons there are three central assumptions. If there is any indication, that these assumptions are violated, no indirect comparison should be carried out at all. The homogeneity assumption is the same as for pairwise meta-analysis. There are already established methods to evaluate this assumption. Second, the similarity assumption, implies that all analyzed 
studies should be comparable (similar) regarding possible effect modifiers across all interventions. This is a qualitative assumption. Methods to evaluate this assumption will always have subjective components, so an objective evaluation will be difficult. There exist some detailed proposals for the evaluation of this assumption, for example by Cope et al. [3]. The assumption of consistency states that the effect estimations from direct and indirect evidence are consistent, meaning that there is no discrepancy between the results of direct and indirect comparisons (that cannot be explained by random error or heterogeneity) [4]. This assumption applies especially for indirect comparisons, which is why new statistical methods to evaluate this assumption have recently been developed or are still under development. However, little research has yet been conducted on their performance.

For simple networks like triangular networks with 3 interventions adjusted indirect comparisons [5] can be conducted. For more complex networks, simultaneous analysis of direct and indirect evidence as well as adequate inclusion of multi-arm studies, a network metaanalysis (NMA) is required. Thus, NMA is becoming increasingly popular in systematic reviews and health technology assessments [6, 7]. However, as well as for methods to evaluate the consistency assumption, there is still ambiguity concerning the properties of effect estimators in NMA. Several unsolved methodological problems [8] lead to a general uncertainty regarding the use and the certainty of results. Moreover, there are no established standards for the practical application.

Therefore we conducted a simulation study. The aim of our simulation study was to investigate the performance of effect estimators in NMA and the evaluation of the consistency assumption. While some simulation studies on NMA already exist, to our knowledge there are hardly any simulation studies analysing complex networks with up to 5 interventions. We also evaluated recently published effect estimators, not evaluated in a simulation study yet, as well as methods to evaluate the consistency assumption in complex networks. On the base of our results, we give recommendations for practical application. This paper is based on a $\mathrm{PhD}$ thesis, which includes all details [9]. An electronic version (in German) is available on request.

This paper is organized as follows. In the "Methods" section, we describe the different estimation approaches and methods to evaluate the consistency assumption. The design of our simulation study is described in the following Section. Subsequently the results of the simulation study are presented and illustrated by an application of a real data example. The paper will be closed with a discussion.

\section{Methods}

The properties of the following effect estimators as well as methods for evaluating the consistency assumption were investigated in our simulation study.

\section{Effect estimators}

Many methods have been proposed for effect estimation in NMA. We focused our investigation on effect estimators for NMA, which can be applied to all kinds of networks and which can handle multi-arm studies properly. Bayesian methods, often called mixed treatment comparisons (MTC), are most commonly used. But recently there has also been a strong focus on frequentist methods. We chose 3 different NMA estimators for our investigation (2 Bayesian, 1 frequentist), which will be described in more detail in the following Sections.

For comparison, we also included 3 direct effect estimators from pairwise meta-analysis. The first one is the frequentist DerSimonian-Laird meta-analysis estimator [10]. Although its shortcomings are now well known, especially with few studies [11], it is still the most frequently used estimator in meta-analysis. It is therefore computed for the purpose of comparison. The second one is the Bayesian meta-analysis estimator [12]. In the following, these estimators will be referred to as $D E_{\text {Frequ }}$ and $\mathrm{DE}_{\text {Bayes }}$ respectively. We also had a look at the estimates from a so called MTC inconsistency model, where no consistency is assumed. Because it is closely related to the MTC consistency effect estimator, the MTC inconsistency model is described in more detail in the "MTC consistency model ( $\left.\mathrm{MTC}_{\mathrm{Con}}\right)$ " section.

For each estimator (direct and NMA) we fitted random effects (consistency) models assuming the same heterogeneity $\tau^{2}$ within each pairwise comparison in the network.

\section{Graph-theoretical approach (netmeta)}

The graph-theoretical approach is a frequentist method, developed by Rücker [13]. Methods from graph theory usually used in electrical networks were transferred to NMA. Briefly, for a network of $n$ interventions and $m$ pairwise comparisons from direct studies a $m \times n$ design matrix $B$ is defined. Let $x_{k}(k=1, \ldots, m)$ denote the observed effects and $V_{k}$ the corresponding variances. Then the $m \times m$ diagonal matrix $W$ contains the inverse variances $\frac{1}{V_{k}}$. With the help of these two matrices a hat matrix $H$ can be estimated by

$$
H=B\left(B^{T} W B\right)^{+} B^{T} W
$$

whereas $\left(B^{T} W B\right)^{+}$is the Moore-Penrose pseudoinverse of the Laplacian matrix $L=B^{T} W B$. Finally, by applying $H$ to the vector of observed effects $x$ consistent weighted least squares effect estimates $\hat{x}_{n m a}$ are established. 
As part of the implementation into the R-package netmeta [14] the originally fixed effect model was extend to a random effects model. The handling of multi-arm studies is described by Rücker und Schwarzer [15]. For the whole model and a more detailed description see also chapter 8 of Schwarzer et al. [16]. In the present article we will refer to this estimator as netmeta.

\section{MTC consistency model ( $\mathrm{MTC}_{\mathrm{Con}}$ )}

The Bayesian MTC consistency model was first introduced by Lu and Ades $[17,18]$. It is a hierarchical model, that combines direct and indirect evidence assuming consistency within a Bayesian framework. Suppose that there $n$ interventions $A, B, C, \ldots$ to be compared in a network. A reference intervention has to be chosen, here denoted as $A$. The effects $d_{A l}$ of all other interventions $l=B, C, \ldots$ with respect to $A$ are modeled directly as basic parameters. Assuming consistency within the network, the effects of all other interventions can then be calculated by $d_{b l}=$ $d_{A l}-d_{A b}$ for $l \neq b, b, l \in\{B, C, \ldots\}$ as functional parameters.

For a binary outcome of study $k$, outcome counts for intervention $l$ are summarized by the number of events $r_{k l}$ out of a number $q_{k l}$ of patients at risk. The number $r_{k l}$ is assumed to follow a binomial distribution with parameters $p_{k l}$ and $q_{k l}$, whereas $p_{k l}$ is modeled by a logit function. For each study $j$, a study specific baseline log-odds $\mu_{k b}$ of reference intervention $b$ is assumed together with the logodds ratio $\delta_{k b l}$ of the outcome for intervention $l$ relative to this study specific reference $b$ :

$$
\begin{aligned}
r_{k l} & \sim \operatorname{Bin}\left(p_{k l}, q_{k l}\right) \\
\operatorname{logit}\left(p_{k l}\right) & = \begin{cases}\mu_{k b} & b=A, B, C, \ldots \text { if } l=b \\
\mu_{k b}+\delta_{k b l} & b=B, C, D, \ldots \text { if } l \text { before } b \text { (in alphabetical order) }\end{cases}
\end{aligned}
$$

In a random effects model we assume the trial-specific $\delta_{k b l} \sim N\left(d_{b l}, \tau^{2}\right)$ to follow a normal distribution with mean log-odds ratio $d_{b l}=d_{A l}-d_{A b}$ and homogeneous variance $\tau^{2}$. For multi-armed trials we consider a multivariate normal distribution with covariance $\frac{\tau^{2}}{2}$ reflecting the assumption of homogeneous variance for all arms. For $\mu_{k b}, d_{b l}$ and $\tau^{2}$ priors have to be established. Due to the lack of prior information we choose non informative priors. The exact specification as well as the initial values of Markov chains can be found in the Online Appendix [see Additional file 1]. For more information as well as exemplary WinBUGS code see Dias et al. [19]. In the present article, we will refer to this estimator as $\mathrm{MTC}_{\mathrm{Con}}$.

As well as a consistency model $\left(\mathrm{MTC}_{\mathrm{Con}}\right)$, an inconsistency model can be fitted. Here, each of the mean relative effects $d_{b l}$ is modelled separately. No consistency is assumed and hence no indirect evidence used. Therefore, this estimator is more a direct estimator than an
NMA estimator and we will refer to it as an direct estimator. Only the variance $\tau^{2}$ will be estimated by all studies in the network collectively instead by one direct comparison alone [20]. In the following we will refer to it as $\mathrm{MTC}_{\text {Incon }}$.

\section{MTC consistency model with stepwise removal of studies contributing to inconsistency identified in a leverage plot $\left(\mathrm{MTC}_{\mathrm{SR}}\right.$ )}

The second Bayesian estimator is also based on the MTC consistency model. Here, all inconsistent studies identified in a leverage plot are removed from the network in a stepwise procedure [21] finally leading to a consistent network. Using the residual deviance approach [22] the study (or study arm for multi-arm studies) contributing most to inconsistency according to the sum of the residual deviance and the leverage, will be eliminated from the analysis and the MTC consistency model will be recalculated. This process is repeated until the network demonstrates no more inconsistency (residual deviance + leverage $\leq 3$ ) [23]. In the present article we will refer to this estimator as $\mathrm{MTC}_{\mathrm{SR}}$.

\section{Evaluating the consistency assumption}

Beside NMA effect estimators, we assessed the corresponding global methods for evaluating the consistency assumption described in the following.

\section{Q statistic from graph-theoretical approach}

The graph-theoretical approach enables the calculation of $Q$ statistics and corresponding $I^{2}$ for the whole network. The extent of variation in the whole network is measured by

$$
Q_{\text {total }}=\left(x-\hat{x}_{n m a}\right)^{T} W\left(x-\hat{x}_{n m a}\right) .
$$

Under the assumptions of homogeneity and consistency $Q_{\text {total }}$ follows a $\chi^{2}$ distribution with $M-(n-1)$ degrees of freedom (df), where $M$ denotes the number of independent studies in the network and $n$ the number of interventions. $Q_{\text {total }}$ can be decomposed into the sum of $k=1, \ldots, K$ statistics for heterogeneity between studies with the same design (set of treatments) in the network $\sum_{k=1}^{K} Q_{h e t_{k}}$ and the remaining design inconsistency $Q_{\text {incon. }}$ So $Q_{\text {incon }}$ can be calculated by

$$
Q_{\text {incon }}=Q_{\text {total }}-\sum_{k=1}^{K} Q_{\text {het }_{k}}
$$

and follows a $\chi^{2}$ distribution with $K-(n-1)$ degrees of freedom. For our simulation study we tested both $Q_{\text {total }}$ and $Q_{\text {incon }}$ with a level of significance of 0.2 . We also calculated the corresponding $I_{\text {total }}^{2}$ and $I_{\text {incon }}^{2}$ by $I^{2}=\left(\frac{Q-d f}{Q}\right) \times$ $100 \%$ and assumed inconsistency if $I^{2}>50 \%$. 


\section{Comparison of MTC consistency and MTC inconsistency model}

We also compared the model fit of the MTC consistency model with an MTC inconsistency model. To assess model fit we used the residual deviance $\operatorname{Dev}_{\text {res }}$ as well as the deviance information criterion DIC [20, 24]. Using the residual deviance we assumed inconsistency if $\operatorname{Dev}_{r e s}$ from the inconsistency model was lower than $\operatorname{Dev}_{r e s}$ from the consistency model. For the DIC we introduced an additional threshold for relevance of 3 [24]. So we only assumed inconsistency if the DIC of the consistency model was more the 3 points higher than the DIC of the inconsistency model.

\section{Stepwise removal of studies contributing to inconsistency identified in a leverage plot}

By means of $\mathrm{MTC}_{\mathrm{SR}}$ we assumed inconsistency when at least one study or study arm was excluded from the network. In the following we will refer to this approach as $S R_{\text {Lev }}$.

\section{Simulation study \\ Simulation scenarios}

We simulated data for 5 different kinds of network sizes and shapes, which are presented in Fig. 1. The straight lines in Fig. 1 indicate direct evidence, whereas the dashed lines indicate the comparison, where inconsistency was introduced in our simulations. We started with a triangular network (a) with 3 interventions $(A, B, C)$ and data for each pairwise comparison. For network (b) we added an intervention $D$ with direct comparisons to intervention $A$ and $C$, but no direct comparisons to intervention $B$. For network (c) we again added another intervention $E$ with direct comparisons to interventions $A$ and $D$. From network (c) to network (d) we did not change the number of interventions, but we added more directs comparisons for intervention $E$ with interventions $B$ and $C$ to the network. In the last network (e) we added an additional inconsistency for comparison $D$ vs. $E\left(R O R_{D E}=0.6\right)$.

In data generation, we introduced inconsistency in the simulated networks by multiplying the consistent odds ratio $(O R)$ with an ratio of odds ratios $(R O R)$, i.e. for comparison $B$ vs. $C$ :

$$
\mathrm{OR}_{B C}^{\text {incon }}=\mathrm{OR}_{B C} \times \mathrm{ROR}_{B C} .
$$

We set a $R O R$ of 1 for no inconsistency, of 0.8 for moderate inconsistency and 0.6 for severe inconsistency. We also simulated a common heterogeneity between the study results in all direct comparisons. To avoid a too strong violation of the homogeneity assumption, we varied heterogeneity by a very small amount only. For a very low degree of heterogeneity we chose $\tau^{2}$ to be 0.01 and for a low degree of heterogeneity we chose 0.1 .

Because of the high computational effort of Bayesian approaches we kept all others parameters fixed. We simulated data of 5 studies for each direct comparison in the network where each study arm contained 100 patients. For the binary endpoint we chose on $O R$ as effect measure with a true treatment effect of 1 in all pairwise comparisons. The baseline risk for intervention $A$ was set to be 0.1 , all simulated studies were 2 -arm studies. For each scenario we conducted $R=1000$ replications. An overview of all simulation input parameters is given in Table 1. (a)

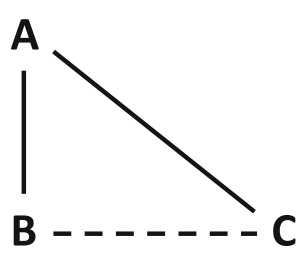

(b)

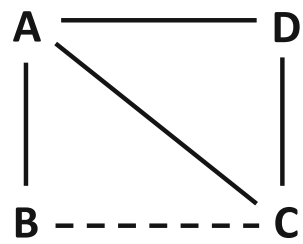

(c)

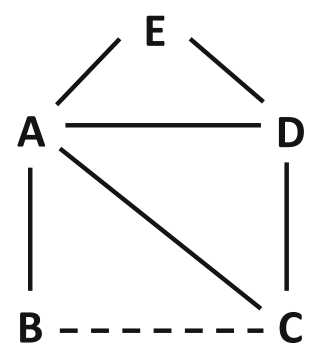

(d)

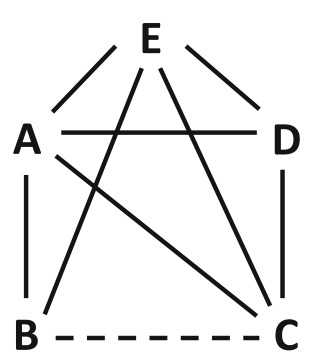

(e)

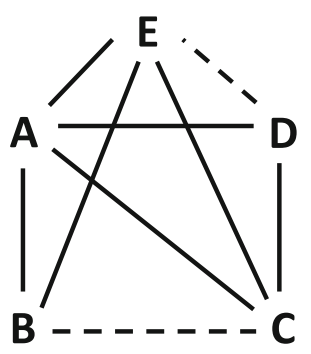

Fig. 1 Graphics of simulated networks. Straight lines indicate direct evidence, dashed lines indicate indirect comparisons with potential inconsistency 
Table 1 Overview of simulated scenarios

\begin{tabular}{ll}
\hline Networks & $\begin{array}{l}\text { (interventions, direct } \\
\text { comparisons) }\end{array}$ \\
\hline Network (a) & $(A, B, C), 3$ \\
Network (b) & $(A, B, C, D), 5$ \\
Network (c) & $(A, B, C, D, E), 7$ \\
Network (d) & $(A, B, C, D, E), 9$ \\
Network (e) & $(A, B, C, D, E), 9$, additional \\
& inconsistency for compari- \\
son $D$ vs. $E$ \\
Inconsistency (OR incon $\left.=\mathrm{OR}_{B C} \times \mathrm{ROR}_{B C}\right)$ & \\
\hline No inconsistency & $\mathrm{ROR} B C=1$ \\
Moderate inconsistency & $\mathrm{ROR} B C=0.8$ \\
Severe inconsistency & $\mathrm{ROR} B C=0.6$ \\
Heterogeneity & \\
\hline Very low heterogeneity & $\tau^{2}=0.01$ \\
Low heterogeneity & $\tau^{2}=0.1$ \\
\hline Direct studies per pairwise comparison & $k=5$ \\
Patients per study arm & $n=100$ \\
True treatment effects & $O R_{A B}=\ldots=O R_{D E}=1.0$ \\
Baseline probability & $p_{A}=0.1$ \\
Replications & $R=1000$ \\
\hline
\end{tabular}

\section{Generation of simulated data}

In the following we will describe the generation of the data for network (d). For all other networks (a) to (c) we simply deleted all not required data leading to the desired network structure. The additional inconsistency in network (e) was similarly to the inconsistency in network (d).

For each pairwise comparison we drew $i=1, \ldots, k$ study-specific log-odds ratios $Y_{i x y}$ from a normal distribution with mean $\log \left(O R_{x y}\right)(x \in\{A, B, C, D\}, y \in$ $\{B, C, D, E\}, x \neq y)$ and variance $\tau^{2}$. For the inconsistent comparison $B$ vs. $C$ we used the mean $\log \left(O R_{B C}^{\text {incon }}\right)$. Using the study-specific $Y_{i x y}$ and the true baseline probabilities $p_{A}$ and $p_{B}=\frac{p_{A} \times O R_{A B}}{1-p_{A} \times\left(1-O R_{A B}\right)}$ we calculated the $i=1, \ldots, k$ study-specific baseline probabilities:

$$
\begin{aligned}
p_{i_{A}}=p_{A}, p_{i_{B}} & =\frac{p_{A} \times \exp \left(Y_{i_{A B}}\right)}{1-p_{A} \times\left(1-\exp \left(Y_{i_{A B}}\right)\right)}, \ldots, p_{i_{E}}=\frac{p_{A} \times \exp \left(Y_{i_{A E}}\right)}{1-p_{A} \times\left(1-\exp \left(Y_{i_{A E}}\right)\right)} \\
\text { and } p_{i_{C}}^{\text {incon }} & =\frac{p_{B} \times \exp \left(Y_{i_{B C}}^{\text {incon }}\right)}{1-p_{B} \times\left(1-\exp \left(Y_{i_{B C}}^{\text {incon }}\right)\right)} .
\end{aligned}
$$

For each study arm the number of events $r_{X}$ was randomly generated assuming a binomial distribution with parameters $n$ and $p_{i_{X}}(X \in\{A, \ldots, E\})$. For all pairwise comparisons including intervention $C$ without inconsistency $p_{i_{C}}$ was used. For the simulated scenarios where inconsistency was introduced for comparison $B$ vs. $C$, $p_{i_{C}}^{\text {incon }}$ was used. If the simulated number of events was 0 in a study arm, we added 0.5 to the cells of the corresponding $2 \times 2$ table.

\section{Performance}

To evaluate the properties of the effect estimators we estimated the coverage probability (CP) of the $95 \%$ confidence or credible intervals by recording the percentage of replications where intervals included the true treatment effect. We also estimated the mean squared error (MSE) by

$$
\widehat{\operatorname{MSE}}(\hat{\theta})=\frac{1}{R} \times \sum_{j=1}^{R}\left(\hat{\theta}_{j}-\theta\right)^{2}
$$

with $\theta$ denoting the true parameter value and $\hat{\theta}_{j}$ the estimated value from replication $j(j=1, \ldots, R)$.

To ease interpretation of results we introduced a classification for the CP represented by a color coding. We classified a CP as good, if it was $\in$ [94\%; 96\%] and as acceptable if it was $\in[90 \% ; 94 \%) \vee(96 \% ; 100 \%]$. A CP below $90 \%$ was classified as not acceptable. We also marked the estimators with the smallest MSE and the second smallest MSE by two or one stars. The actual values of the MSE for all effect estimators can be found in the Online Appendix [see Additional file 1]. An estimator with good properties should optimize the MSE under the side condition of an adequate $\mathrm{CP}$.

For the methods to evaluate the consistency assumption we calculated the percentage of the correct and false decisions for inconsistency.

Again, we introduced a color coding to ease interpretation of results. In the cases, where inconsistency was present in our simulated data sets, we set the proportion of replications, in which a good approach should identify inconsistency to at least $90 \%$. We categorized an approach as acceptable if this was the case in at least $75 \%$ of the replications. Below 75\% we categorized the properties of the approach as not acceptable anymore. In the cases with no inconsistency in the data set, we set the cut-offs for good approach by a maximum of $5 \%$ of replications with identified inconsistency, an acceptable approach by a maximum of $25 \%$ and with more than $25 \%$ we categorized it as not acceptable anymore.

\section{Software implementation}

We run the simulation study in the freely available software R 2.14.1 [25]. For the frequentist DerSimonian-Laird meta-analysis estimator we used the $\mathrm{R}$ package metafor (version 1.6-0) [26]. We implemented the Bayesian models by Markov chain Monte Carlo (MCMC) methodology into OpenBUGS (version 3.0.3) [27], which we called from $\mathrm{R}$ with the package BRugs (version 0.5-3) [28] using the example code provided by Dias et al. [19]. For each replication we used 3 chains with a burn-in of 20000 iterations followed by 40000 updates to obtain posterior estimates. 
Convergence was assessed by the Brooks-Gelman-Rubin method [29] and by visual inspection of the history plots of random samples. For trial baselines and basic parameters vague priors were specified.

The estimations for the graph-theoretical approach were calculated with the R package netmeta (version 0.31 ), which required a newer $R$ version 3.0.2 [30]. The OpenBUGS code for the Bayesian models including the specification of the non informative prior distributions and the starting values for the 3 Markov chains is given in the Online Appendix [see Additional file 1].

\section{Results}

For the evaluated effect estimators as well as the methods to evaluate the consistency assumption we focused our analysis on the 3 comparisons, which were available in all simulated networks: comparison $B$ vs. $C$, where inconsistency was introduced, and the comparisons $A$ vs. $B$ and $A$ vs. $C$. Together, all 3 form the closed loop $A B C$.

\section{Effect estimators}

Table 2 shows the CP for all evaluated effect estimators and scenarios for comparison $B$ vs. $C$ that includes inconsistency in the simulated networks.

The first obvious result of Table 2 is, that in scenarios with severe inconsistency $\left(R O R_{B C}=0.6\right)$ non of the evaluated effect estimators showed an acceptable $C P$ for comparison $B$ vs. $C$. $\mathrm{DE}_{\mathrm{Frequ}}$ showed good $\mathrm{CP}$ for the scenarios with no inconsistency, but no acceptable CP for the scenarios with a moderate degree of inconsistency. The $C P$ of $D E_{\text {Bayes }}$ for these scenarios was acceptable but always too high with values between $97.5 \%$ and $99.6 \%$. $\mathrm{MTC}_{\text {Incon }}$ had no acceptable CP for all but one of the scenarios with moderate inconsistency. For the consistent scenarios its $\mathrm{CP}$ was at least acceptable, but slightly worse than the one from $\mathrm{DE}_{\mathrm{Frequ}}$. Out of all the NMA estimators $\mathrm{MTC}_{\mathrm{SR}}$ showed the worst CP for all scenarios. The two remaining NMA estimators $\mathrm{MTC}_{\mathrm{Con}}$ and netmeta had a not acceptable $\mathrm{CP}$ in the case of moderate inconsistency and low heterogeneity. With moderate inconsistency and very low inconsistency however both showed an acceptable CP with one exception for $\mathrm{MTC}_{\mathrm{Con}}$. In the consistent scenarios $\mathrm{MTC}_{\mathrm{Con}}$ and netmeta had both at least acceptable $\mathrm{CP}$ with the exception of network (d) with low heterogeneity, where the CP for both estimators was not acceptable anymore. Concerning the MSE netmeta showed the smallest MSE for all scenarios, whereas $\mathrm{MTC}_{\mathrm{Con}}$ had always the second smallest MSE.

The CP and the MSE for the comparisons $A$ vs. $B$ and $A$ vs. $C$ can be found in the Online Appendix [see Additional file 1]. As for these comparisons no inconsistency was introduced, all direct estimators showed at least acceptable coverage probabilities as it was to be expected. However, the network estimators use the information from the potential inconsistent comparison $B$ vs. $C$ also for these comparisons. Therefore their results are more interesting. For most of the simulated scenarios $\mathrm{MTC}_{\mathrm{Con}}$ as well as netmeta showed acceptable and often even good CP. All exceptions for both estimators lay in the scenarios with severe inconsistency. $\mathrm{MTC}_{\mathrm{SR}}$ however showed not acceptable $C P$ in most of the scenarios. Also for the two comparisons $A$ vs. $B$ and $A$ vs. $C$ netmeta had the smallest and $\mathrm{MTC}_{\mathrm{Con}}$ the second smallest MSE with few exceptions.

Additionally the simulation study showed a low dependency of the properties of the effect estimators on the network size. Especially for NMA estimators, validity of the homogeneity assumption is central, therefore its verification is crucial. Inclusion of additional studies is to be preferred over the inclusion of additional interventions.

\section{Evaluating the consistency assumption}

Table 3 presents the results of the methods for evaluating the consistency assumption.

The proportion of replications with a wrong decision for inconsistency for $\mathrm{Dev}_{\text {res }}$ and $\mathrm{SR}_{\mathrm{Lev}}$ was not acceptable with values ranging from $37.8 \%$ to $66.1 \%$ and from $32.2 \%$ to $87.8 \%$ respectively. $Q_{\text {incon }}$ and $I_{\text {incon }}^{2}$ showed slightly better results under consistency with values between $15.2 \%$ $22.8 \%$ and $5.3 \%-17.9 \%$, which we categorized as acceptable. Concerning the wrong decision for inconsistency $Q_{\text {total }}$ and $I_{\text {total }}^{2}$ were highly dependable on the underlying heterogeneity. With very low heterogeneity in the networks $\left(\tau^{2}=0.01\right)$ both showed low proportions of wrong decisions $(\leq 5 \%)$, but with low heterogeneity $\left(\tau^{2}=0.1\right)$ in the networks $I_{\text {total }}^{2}$ led to wrong decisions in $6.1 \%$ to $14.3 \%$ of the replications and $Q_{\text {total }}$ in $28.5 \%$ to $58.5 \%$. Only DIC showed good properties concerning the proportions of wrong decisions for inconsistency with only $0.7 \%$ to $2.5 \%$ in all scenarios with consistency. However, in the scenarios, where inconsistency was present, DIC indicated inconsistency only in a few replications as well $(1.2 \%$ to $28.3 \%$ ), which we categorized as not acceptable. Dev res already showed high proportions of decisions for inconsistency in the scenarios with consistency, these values increased just slightly for the scenarios with inconsistency (43.7\% to $84.5 \%)$ and were categorized as acceptable in only 2 scenarios (network (e), very low heterogeneity, moderate and severe inconsistency). All 4 methods for evaluating the consistency based on netmeta showed no acceptable proportions of decisions for inconsistency in any of the simulated scenarios with inconsistency. The values for $Q_{\text {total }}$ ranged between $4.2 \%$ and $70.0 \%$ and for $I_{\text {total }}^{2}$ between $0 \%$ and $16.5 \%$. It is remarkable however, that both methods showed noticeably higher proportions in the scenarios with low heterogeneity than the corresponding ones with very low heterogeneity. $Q_{\text {incon }}$ and 
Table 2 Combination of estimated coverage probabilities [in \%] and MSE for comparison B vs. C

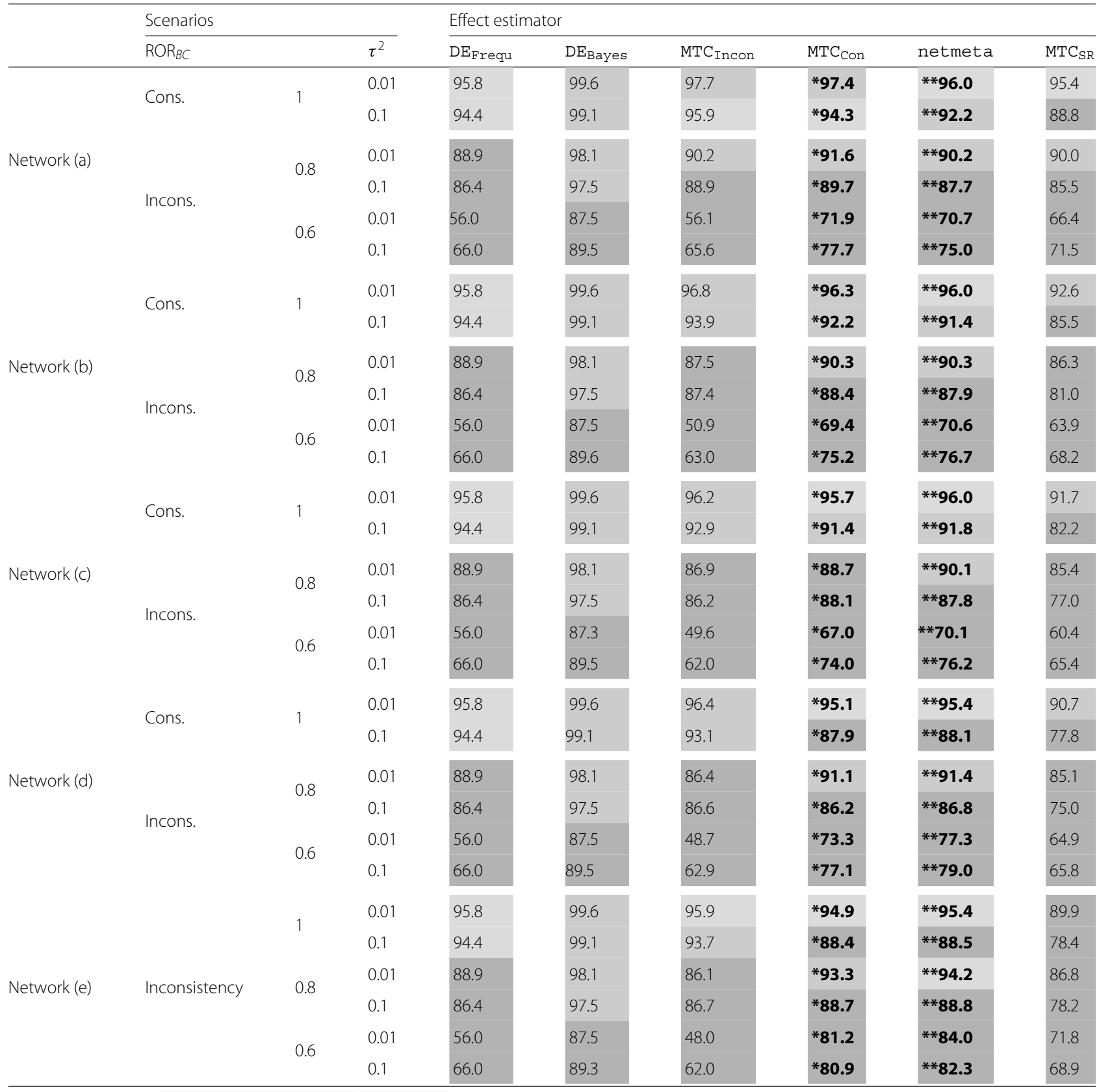

Coding: $\in[94 \% ; 96 \%] \in[90 \% ; 94 \%) \vee(96 \% ; 100 \%] \in[0 \% ; 90 \%)$

** Smallest MSE

*Second smallest MSE

$I_{\text {incon }}^{2}$ indicated inconsistency in $20.1 \%$ to $62.8 \%$ and $6.5 \%$ to $42.5 \%$ of the replications for the scenarios with inconsistency respectively. $\mathrm{SR}_{\mathrm{Lev}}$ already showed the highest proportions of decisions for inconsistency in the scenarios with consistency. These proportions increased, when inconsistency was present to values between $30.7 \%$ and 92.3\%. With one exception (network (c), very low heterogeneity, severe inconsistency) these proportions were categorized as at least acceptable for the bigger networks (c), (d) and (e).

Overall we found that none of the evaluated methods reliably identified inconsistency and the identification of inconsistency just slightly depended of true underlying inconsistency. For some methods like the comparison of the consistency and the inconsistency MTC model by means of the $\operatorname{Dev}_{r e s}$ and the stepwise removal of studies 
Table 3 Proportion of replications with a decision for inconsistency[in \%]

\begin{tabular}{|c|c|c|c|c|c|c|c|c|c|c|}
\hline & Scenarios & & & Approa & & & & & & \\
\hline & $\overline{\mathrm{ROR}_{B C}}$ & & & MTC & & netm & & & & SR \\
\hline & & & $\tau^{2}$ & $\mathrm{DEV}_{\text {res }}$ & DIC & $Q_{\text {total }}$ & $R_{\text {total }}^{2}$ & Qincon & $R_{\text {incon }}^{2}$ & Lev \\
\hline & Conc & 1 & 0.01 & 37.8 & 1.3 & 3.9 & 0.8 & 18.8 & 14.8 & 32.2 \\
\hline & . & & 0.1 & 50.0 & 1.3 & 28.5 & 14.3 & 22.8 & 17.9 & 34.2 \\
\hline Network (a) & & 08 & 0.01 & 43.7 & 2.5 & 4.2 & 1.2 & 26.4 & 22.4 & 30.7 \\
\hline & Inconc & & 0.1 & 50.2 & 1.8 & 26.8 & 13.8 & 28.6 & 23.8 & 35.6 \\
\hline & . & 06 & 0.01 & 61.3 & 9.3 & 9.1 & 2.9 & 46.7 & 40.2 & 33.0 \\
\hline & & & 0.1 & 59.0 & 6.4 & 30.8 & 16.5 & 46.1 & 41.6 & 35.2 \\
\hline & Cons & 1 & 0.01 & 41.0 & 1.8 & 3.9 & 0.3 & 15.7 & 10.8 & 58.7 \\
\hline & & & 0.1 & 56.1 & 1.0 & 40.2 & 10.6 & 19.1 & 13.4 & 59.1 \\
\hline Network (b) & & 08 & 0.01 & 44.7 & 3.3 & 4.6 & 0.3 & 20.7 & 16.1 & 55.8 \\
\hline & Incons & & 0.1 & 54.0 & 1.4 & 38.3 & 10.6 & 24.8 & 17.5 & 61.1 \\
\hline & 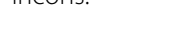 & 06 & 0.01 & 62.5 & 10.3 & 8.4 & 0.4 & 42.0 & 33.5 & 60.6 \\
\hline & & & 0.1 & 62.4 & 5.3 & 42.5 & 11.1 & 41.7 & 33.7 & 59.5 \\
\hline & Cons & 1 & 0.01 & 46.0 & 2.5 & 4.7 & 0.1 & 16.7 & 8.9 & 75.1 \\
\hline & & & 0.1 & 62.5 & 0.9 & 47.9 & 7.3 & 17.1 & 11.4 & 73.1 \\
\hline Network (c) & & 08 & 0.01 & 47.2 & 2.8 & 4.7 & 0.1 & 21.8 & 12.0 & 75.3 \\
\hline & Incons & 0.0 & 0.1 & 61.8 & 1.3 & 46.2 & 7.8 & 22.2 & 12.9 & 77.1 \\
\hline & & 06 & 0.01 & 63.4 & 10.2 & 6.9 & 0.1 & 37.3 & 25.4 & 77.8 \\
\hline & & & 0.1 & 63.0 & 4.0 & 50.1 & 8.7 & 36.3 & 25.3 & 73.3 \\
\hline & Cons & 1 & 0.01 & 53.7 & 2.5 & 4.9 & 0.1 & 15.2 & 6.0 & 87.8 \\
\hline & 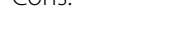 & & 0.1 & 66.1 & 0.7 & 58.5 & 6.1 & 16.5 & 5.3 & 81.9 \\
\hline Network (d) & & 08 & 0.01 & 56.7 & 2.6 & 4.3 & 0.0 & 20.1 & 6.5 & 87.8 \\
\hline & Inconc & & 0.1 & 69.3 & 1.2 & 58.9 & 6.7 & 22.2 & 9.2 & 82.5 \\
\hline & & 06 & 0.01 & 71.9 & 12.4 & 6.9 & 0.0 & 37.4 & 19.9 & 90.5 \\
\hline & & & 0.1 & 69.9 & 2.9 & 63.1 & 8.5 & 35.3 & 18.0 & 79.6 \\
\hline & & 1 & 0.01 & 71.5 & 13.0 & 9.0 & 0.0 & 41.4 & 21.9 & 91.4 \\
\hline & & & 0.1 & 67.9 & 3.9 & 64.6 & 7.2 & 43.1 & 24.0 & 79.9 \\
\hline Network (e) & Inconsistency & 08 & 0.01 & 77.7 & 14.4 & 9.0 & 0.0 & 45.0 & 23.9 & 88.9 \\
\hline & & & 0.1 & 69.5 & 5.9 & 64.6 & 8.0 & 47.9 & 29.6 & 80.6 \\
\hline & & 06 & 0.01 & 84.5 & 28.3 & 12.9 & 0.0 & 62.8 & 42.5 & 92.3 \\
\hline & & & 0.1 & 69.7 & 7.8 & 70.0 & 10.6 & 60.8 & 41.5 & 78.2 \\
\hline
\end{tabular}

Coding with consistency: $\in[0 \% ; 5 \%] \in(5 \% ; 25 \%] \in(25 \% ; 100 \%]$

Coding with inconsistency: $\in[90 \% ; 100 \%] \in[75 \% ; 90 \%) \in[0 \% ; 75 \%)$

contributing to inconsistency identified by a leverage plot the proportion of identified inconsistencies was relatively high. In contrast, for most methods, this proportion was rather small, independently to the underlying truth.

\section{Real data example}

To illustrate the application of the different effect estimators as well as methods for evaluating the consistency assumption we applied them to a real data example.
The data example is based on two previous benefit assessments for the treatment of depression carried out by the Institute for Quality and Efficiency in Health Care (IQWiG) [31, 32], which was also used by Sturtz and Bender [23]. We used the results for the outcome response to treatment. We had data for 7 different individual drugs, 2 drug classes and placebo. Because the data are taken from two previous benefit assessments it can be assumed that the similarity assumption is met at least roughly. 
We abandoned further investigation of this assumption because the objective of this example was purely methodological and no clinical conclusions should be made. The evaluation of the homogeneity assumption led to a exclusion of 3 two-arm studies and 1 arm from a three-arm study. This led to a final data pool of 100 studies (75 two-arm studies, 25 three-arm studies) for the comparison of the 10 treatments. For the possible 45 pairwise comparisons between all treatments, there were headto-head studies for 21 of them. The network is shown in Fig. 2.

\section{Effect estimators}

The results for the different evaluated effect estimators for the antidepressants network are presented in the Online Appendix [see Additional file 1]. Overall the results from netmeta and $\mathrm{MTC}_{\mathrm{Con}}$ were very similar. With the exception of one comparison (tri- and tetracyclic antidepressants vs. placebo) both estimates agreed in statistical significance, point estimates and confidence/credible intervals. However, $\mathrm{MTC}_{\mathrm{SR}}$ provided much more frequently statistically significant results. The direct estimator $\mathrm{DE}_{\text {Bayes }}$ turned out to be not suitable due to very wide credible intervals. Additionally, the results illustrated one of the main advantages of network meta analysis, which was not as obvious in the simulation study. While the direct estimators can only provide effect estimates if head to head studies are available, network meta analysis estimators provide estimates for all possible comparisons in the network.

\section{Evaluating the consistency assumption}

For evaluating the consistency assumption by comparing the MTC consistency and the MTC inconsistency model the two models were fit to the data and the DIC and the residual deviance were calculated. The DIC was 373.1 for the inconsistency model and 370.1 for the consistency model whereas the residual deviance was 244.5 for the inconsistency model and 237.7 for the consistency model. Thus both measures for model fit favored the consistency model and identified no sign of global inconsistency. (It should be noted that, with 225 data points in the network the consistency model had not an optimal model fit as well.) Likewise the different evaluated methods for evaluating the consistency assumption from the software package netmeta were applied

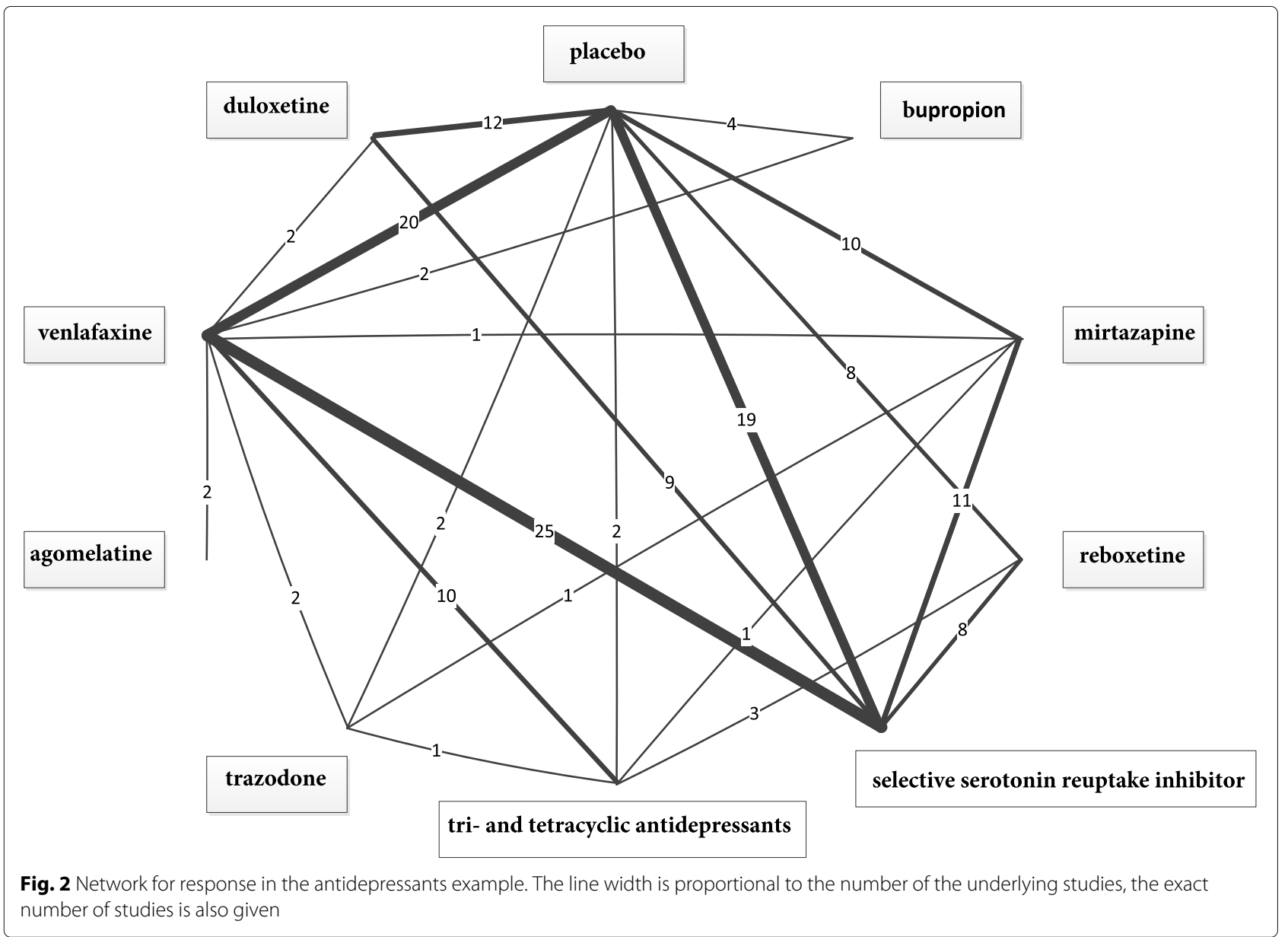


to the data example. For $I_{\text {total }}^{2}$ and $I_{\text {incon }}^{2}$ this resulted in values of $23.5 \%$ and $8.3 \%$. Comparing them with a cut-off of $50 \%$, both statistics showed no global inconsistency. The same holds for Qincon. The corresponding p-value was 0.637 and hence not statistically significant at a predefined level of significance of 0.2 . Only $\mathrm{Q}_{\text {total }}$ identified global inconsistency with a corresponding p-value of 0.015. $\mathrm{MTC}_{\mathrm{SR}}$ identified inconsistency in the network with a total of 4 inconsistent 2 -arm studies and 4 inconsistent arms of 3-arm studies. In Table 4 an overview of the results of the different methods to evaluate the consistency assumption in the antidepressants example is given.

The evaluated methods showed different results for the global inconsistency. Five methods identified no inconsistency, whereas two $\left(Q_{\text {total }}\right.$ and $\left.S R_{L e v}\right)$ identified inconsistency.

\section{Discussion}

In this paper, we presented a simulation study aimed to investigate the properties of different effect estimators and methods to evaluate the consistency assumption in NMA. The results of our study indicated that with moderate or no inconsistency and very low heterogeneity the estimator from a Bayesian MTC consistency model $\mathrm{MTC}_{\mathrm{Con}}$ and the estimator from the graph-theoretical approach by the R-package netmeta showed acceptable properties concerning a coverage probability of $90 \%$ and higher and a relatively small MSE, whereas netmeta had slightly better properties. However, none of the evaluated effect estimators showed acceptable properties for networks with a high degree of inconsistency. Coverage probabilities for $\mathrm{MTC}_{\mathrm{Con}}$ and netmet a ranged from $67.0 \%$ to $81.2 \%$ $\left(\mathrm{MTC}_{\mathrm{Con}}\right)$ and from $70.1 \%$ to $84.0 \%$ (netmeta), respectively. So, there is a strong need to evaluate and ensure the consistency assumption to get effect estimates with acceptable properties. If the consistency assumption is seriously violated, no NMA should be carried out at all. However, concerning the evaluated methods to evaluate the consistency assumption, none were shown to be suitable. This a huge problem for the application of NMA in practice. All evaluated methods showed either high or low proportions of replications with a decision for inconsistency in all scenarios independently of the underlying true consistency. The comparison of the MTC consistency

Table 4 Overview of the identification of global inconsistency in the antidepressants example for all evaluated methods

\begin{tabular}{|c|c|c|c|c|c|c|}
\hline \multicolumn{2}{|l|}{ MTC } & \multicolumn{4}{|c|}{ netmeta } & \multirow{2}{*}{$\begin{array}{l}\text { SR } \\
\text { Lev }\end{array}$} \\
\hline $\mathrm{DEV}_{\text {res }}$ & DIC & $\mathrm{Q}_{\text {total }}$ & $P_{\text {total }}^{2}$ & Qincon & $l_{\text {incon }}^{2}$ & \\
\hline- & - & + & - & - & - & + \\
\hline
\end{tabular}

and MTC inconsistency model by the residual deviance $\operatorname{Dev}_{\text {res }}(37.8 \%-84.5 \%)$ and the stepwise removal of studies contributing to inconsistency identified in a leverage plot $\mathrm{MTC}_{\mathrm{SR}}(30.7 \%-92.3 \%)$ showed in all scenarios rather high proportions, which increased with network size. For the DIC the proportions were always small with values up to a maximum of $28.3 \%$ and slightly higher proportions in scenarios with very low heterogeneity. The methods based on the graph-theoretical approach measuring the extent of the variation in the whole network $Q_{\text {total }}$ and $I_{\text {total }}^{2}$ showed higher proportions of decisions for inconsistency in all scenarios with low heterogeneity. This was particularly pronounced for $Q_{\text {total }}$. For example in network (d) with a high degree of inconsistency and very low heterogeneity there were only $6.9 \%$ correct decisions for inconsistency, but with low heterogeneity this proportion increased to $63.1 \%$ even though the same amount of inconsistency was present in the generated data set. The findings suggest, that these methods detect rather heterogeneity not inconsistency. The methods based on the graph-theoretical approach measuring the extent of the variation in the network caused by inconsistency $Q_{\text {incon }}$ and $I_{\text {incon }}^{2}$ both led to acceptable proportions of wrong decisions for inconsistency with values between $15.2 \%$ and $22.8 \%$ and $5.3 \%$ and $17.9 \%$ respectively. However, in the scenarios with underlying inconsistency both methods detected inconsistency only in a maximum of $62.8 \%$ of the replications. This means that the proportion of detected inconsistencies did just slightly differ between the different scenarios with or without true inconsistency for all methods. We also saw a dependency to the amount of heterogeneity and only a low impact of the network size, whereas better properties for a network with more studies than with more interventions were observed.

Different aspects of the properties of effect estimators and methods to evaluate the consistency assumption in NMA have also been evaluated in other simulation studies [33-38]. However, most of the existing simulation studies evaluated the adjusted indirect comparison according to Bucher [5]. Song et al. [36] were the first ones, who also evaluated the Bayesian MTC effect estimator as well as methods to evaluate the consistency assumption. Similar to our study, they found that all effect estimators provided unbiased results, when no inconsistency was present. For the methods to evaluate the consistency assumption the power to detect inconsistencies was very small. Even with 120 studies the maximum power was about $70 \%$. Jonas et al. [37] evaluated the properties of the Bayesian MTC effect estimator for the probability to be the best intervention in networks with up to 4 interventions and found only little influence of the number of studies (2 to 10) per pairwise comparison in the network. Veroniki et al. [38] evaluated the influence of different network properties on the estimation of inconsistency in a network of 
3 interventions by the difference of the direct estimation and the adjusted indirect estimation according to Bucher [5]. As well as Song et al. [36] they found, that the test had low power, wheres the power was slightly higher when estimating the heterogeneity variance for the pairwise comparisons with the method by Knapp and Hartung $[39,40]$. The low power was similar to the findings in our simulation study for the more complex methods to evaluate the consistency assumption for networks with up to 5 interventions.

Most simulation studies regarding NMA consider only networks with 4 or less interventions. We conducted a simulation study for networks with up to 5 interventions. Moreover the evaluated effect estimators for NMA in our simulation study differ from previous simulation studies with the majority evaluating the adjusted indirect comparison according to Bucher [5]. However, for more complex network structures this simple approach is not possible. The estimator netmeta as well as the estimator based on a MTC consistency model with stepwise removal of studies contributing to inconsistency identified in a leverage plot $\mathrm{MTC}_{\mathrm{SR}}$ have never been evaluated before. Until now, there also have only been few studies, which evaluated methods to evaluate the consistency assumption at all and most of them compared direct and indirect evidence in a very simple way [36, 38]. Again, for more complex networks this approach is not possible.

Because of the computational intensity of the Bayesian MCMC methods, only a limited number of scenarios could be considered in this simulation study. In addition to the network size, consistency and heterogeneity, the numbers of studies per pairwise comparison, the sample size in the studies, the true underlying effects, the baseline probability and other effect measures would be interesting to assess. For ease of implementation only two-arm studies were simulated. Since all evaluated approaches can handle multi-arm studies properly, the impact of multiarm studies should also be a topic of further research. In the simulated data as well as in the models underlying the NMA estimators the same heterogeneity $\tau^{2}$ was assumed in each pairwise comparison. This homogeneous variance structure has already been questioned by others and should be further evaluated. Thorlund et al. [41] for example suggest for the Bayesian context the use of informative priors instead.

Another result of our study is that definitely further evaluation of the approaches in NMA is needed. Especially reliable methods to evaluate the consistency assumption in complex networks are missing and future research should focus on that topic. We also evaluated only global methods to evaluate the consistency assumption. A further evaluation of local methods like node-splitting [22] and the newly proposed composite likelihood method [42] could be helpful in detecting inconsistency and dealing with it. Another way to deal with the current lack of reliable methods to evaluate the consistency assumption could be the use of estimators, which can handle a higher degree of inconsistency, like models with inconsistency parameters [43-48]. The properties of these estimators should also be investigated in further simulation studies. Furthermore it is a problem for the methods to evaluate the consistency assumption to distinguish between heterogeneity and inconsistency. This is another important topic, where more research is required.

After the implementation of this simulation study, there has been the development of a user-friendly $\mathrm{R}$ package gemtc [49] for conducting Bayesian network meta-anaylsis using JAGS (Just another Gibbs sampler) with several useful features. Users, more familiar with the software $R$, can use this package instead of the BUGS software. For a guide to the practical application of gemtc as well as netmeta see also [50].

$$
\begin{aligned}
& \text { - Have a strong focus on the evaluation of the similarity assumption: } \\
& \text { - Check priorly defined important study and patient characteristics thoroughly. } \\
& \text { - Be rather rigorous by evaluating their comparability. } \\
& \text { - Evaluate the homogeneity assumption: } \\
& \text { - If any violations are detected, check again for differences in study and patient } \\
& \text { characteristics. } \\
& \text { - Use either } \text { MTC Con }_{\text {or netmeta for NMA. }} \\
& \text { If there are any concerns regarding the satisfaction of the central assumptions of NMA, } \\
& \text { no NMA should be carried out at all! }
\end{aligned}
$$

Fig. 3 Recommended pragmatic approach 


\section{Conclusions}

According to the results of our simulation study we recommend a pragmatic approach as currently best possible procedure for practical application in NMA, which is shown in Fig. 3. The estimators netmeta or $\mathrm{MTC}_{\mathrm{Con}}$ showed the best properties concerning coverage probability and mean squared error and therefore should be used. Since none of the evaluated methods for checking the consistency assumption showed acceptable properties, there should be a strong focus on the evaluation of the similarity assumption and one should rather be rigorous by evaluating it, since it is currently the only way to avoid a high risk of inconsistency in the network as well. Important study and patient characteristics for the investigated research question should be defined a priori and the studies included in NMA should be comparable regarding these characteristics. It is also very important to evaluate the homogeneity assumption and make sure, there are no violations. If violations are detected by a statistical test for homogeneity, the studies should be checked again for differences in their characteristics that can potentially explain the heterogeneity. If there are any concerns regarding the satisfaction of these central assumptions of NMA, no NMA should be carried out at all. Additionally, networks with more studies over networks with more interventions should be preferred.

\section{Supplementary information}

Supplementary information accompanies this paper at

https://doi.org/10.1186/s12874-020-0917-3.

Additional file 1: Online appendix. Additional tables and OpenBUGS Code for the Bayesian models including the specification of the non informative prior distributions.

\section{Abbreviations}

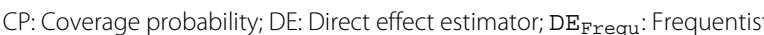
direct effect estimator; $\mathrm{DE}_{\text {Bayes }}$ : Bayesian direct effect estimator; $\mathrm{Dev}_{\text {res: }}$ : Residual deviance; DIC: Deviance information criterion; Lev: Leverage; MCMC Markov chain Monte Carlo; MSE: Mean squared error; MTC: Mixed treatment comparison; $\mathrm{MTC}_{\mathrm{Con}}$ : MTC consistency model; $\mathrm{MTC}_{\text {Incon }}$ : MTC inconsistency model; $\mathrm{MTC}_{\mathrm{SR}}$ : MTC consistency model with stepwise removal of studies; NMA: Network meta-analysis; OR: Odds ratio; ROR: Ratio of odds ratios; $\mathrm{SR}_{\text {Lev }}$ : Stepwise removal of studies by means of the leverage plot

\section{Acknowledgements}

None

\section{Authors' contributions}

CK and RB conceived the concept of this simulation study. CK carried out the simulations and drafted the first version of the manuscript. SS critically reviewed and made substantial contributions to the manuscript. All authors commented on and approved the final manuscript.

\section{Funding}

Not applicable

\section{Availability of data and materials}

The data are available in Kiefer [9] via the German National Library of Medicine (ZB MED) in Cologne (Database: Catalogue ZB MED Medicine, Health; 38 M K: ZB MED, Shelf mark: 2016 D 392).
Ethics approval and consent to participate

Not applicable

\section{Consent for publication}

Not applicable

\section{Competing interests}

The authors declare that they have no competing interests.

Received: 13 August 2019 Accepted: 30 January 2020

Published online: 24 February 2020

\section{References}

1. Salanti G, Schmid CH. Research Synthesis Methods special issue on network meta-analysis: introduction from the editors. Res Synth Meth. 2012;3:69-70.

2. Salanti G. Indirect and mixed-treatment comparison, network, or multipletreatments meta-analysis: many names, many benefits, many concerns for the next generation evidence synthesis tool. Res Synth Meth. 2012;3:80-97.

3. Cope S, Zhang J, Saletan S, Smiechowski B, Jansen JP, Schmid P. A process for assessing the feasibility of a network meta-analysis: a case study of everolimus in combination with hormonal therapy versus chemotherapy for advanced breast cancer. BMC Med. 2014;12:93.

4. Schöttker B, Lühmann D, Boulkhmair D, Raspe H. Indirekte Vergleiche von Therapieverfahren. Köln: Band 88, Schriftenreihe Health Technology Assessment (HTA) in der Bundesrepublik Deutschland; 2009.

5. Bucher $\mathrm{HC}$, Guyatt GH, Griffith LE, Walter SD. The results of direct and indirect treatment comparisons in meta-analysis of randomized controlled trials. J Clin Epidemiol. 1997;50:683-91.

6. Lee AW. Review of mixed treatment comparisons in published systematic reviews shows marked increase since 2009. J Clin Epidemiol. 2014;67: 138-43.

7. Petropoulou M, Nikolakopoulou A, Veroniki AA, Rios P, Vafaei A, Zarin W Giannatsi M, Sullivan S, Tricco AC, Chaimani A, Egger M, Salanti G. Bibliographic study showed improving statistical methodology of network meta-analyses published between 1999 and 2015. J Clin Epidemiol. 2017:82:20-8.

8. Song F, Loke YK, Walsh T, Glenny AM, Eastwood AJ, Altman DG Methodological problems in the use of indirect comparisons for evaluating healthcare interventions: survey of published systematic reviews. BMJ. 2009;338:b1147

9. Kiefer C. Netzwerk Meta-Analyse Schätzer und die Untersuchung der Konsistenzannahme - Ein Vergleich verschiedener Verfahren. PhD thesis. Cologne: University of Cologne; 2015. https://www.ub.uni-koeln.de/ usbportal?query=zbmed:994558.

10. DerSimonian R, Laird NM. Meta-analysis in clinical trials. Control Clin Trials. 1986;7:177-88.

11. IntHout J, loannidis JP, Borm GF. The Hartung-Knapp-Sidik-Jonkman method for random effects meta-analysis is straightforward and considerably outperforms the standard DerSimonian-Laird method. BMC Med Res Methodol. 2014:14:25.

12. Welton NJ, Sutton AJ, Cooper NJ, Abrams KR, Ades AE. Evidence Synthesis for Decision Making in Healthcare. Ltd: Chichester: Wiley; 2012

13. Rücker G. Network meta-analysis, electrical networks and graph theory. Res Synth Meth. 2012;3:312-24.

14. Rücker G, Schwarzer G. netmeta: network meta-analysis with R. R package version 0.3-1. 2013. http://CRAN.R-project.org/package=netmeta. Accessed 05 Aug 2013.

15. Rücker G, Schwarzer G. Reduce dimension or reduce weights? Comparing two approaches to multi-arm studies in network meta-analysis. Stat Med. 2014;33:4353-69.

16. Schwarzer G, Carpenter JR, Rücker G. Meta-Analysis with R. Basel: Springer International Publishing; 2015

17. Lu G, Ades AE. Combination of direct and indirect evidence in mixed treatment comparisons. Stat Med. 2004:23:3105-24

18. Lu G, Ades AE. Assessing evidence inconsistency in mixed treatment comparisons. J Am Stat Assoc. 2006;101:447-59.

19. Dias S, Welton NJ, Sutton AJ, Ades AE. NICE DSU Technical Support Document 2: A Generalised Linear Modelling Framework for Pairwise and Network Meta-Analysis of Randomised Controlled Trials. 2011. last updated September 2016. http://www.nicedsu.org.uk. Accessed 13 Oct 2016. 
20. Dias S, Welton NJ, Sutton AJ, Caldwell DM, Lu G, Ades AE. NICE DSU Technical Support Document: 4 Inconsistency in Networks of Evidence Based Upon Randomised Controlled Trials. 2011. http://www.nicedsu.org. uk. Accessed 13 Oct 2016.

21. Reken S, Sturtz S, Kiefer C, Böhler YB, Wieseler B. Assumptions of Mixed Treatment Comparisons in Health Technology Assessments - Challenges and Possible Steps for Practical Application. PLoS One. 2016;11:e0160712.

22. Dias S, Welton NJ, Caldwell DM, Ades AE. Checking consistency in mixed treatment comparison meta-analysis. Stat Med. 2010;29:932-44.

23. Sturtz S, Bender R. Unsolved issues of mixed treatment comparison meta-analysis: network size and inconsistency. Res Synth Meth. 2012;3: 300-11.

24. Spiegelhalter DJ, Best NG, Carlin BP, van der Linde A. Bayesian measures of model compelxity and fit. J R Stat Soc Ser B (Stat Methodol). 2002;64: 583-639.

25. R Development Core Team. R: A language and environment for statistical computing. Vienna: R Foundation for Statistical Computing; 2011. http:// www.R-project.org/.

26. Viechtbauer $\mathrm{W}$. Conducting meta-analyses in $\mathrm{R}$ with the metafor package J Stat Softw. 2010;36:1-48.

27. Lunn D, Spiegelhalter D, Thomas A, Best N. The BUGS project: evolution, critique and future directions. Stat Med. 2009;28:3049-67.

28. Thomas A, O'Hara B, Ligges U, Sturtz S. Making BUGS Open. R News. 2006:6:12-7.

29. Brooks SP, Gelman A. General methods for monitoring convergence of iterative simulations. J Comput Graph Stat. 1998;7:434-55.

30. R Core Team. R: A language and environment for statistical computing. Vienna: R Foundation for Statistical Computing; 2013. http://www.Rproject.org/.

31. IQWiG. Selektive Serotonin- und und

Noradrenalin-Wiederaufnahmehemmer (SNRI) bei Patienten mit Depressionen. Abschlussbericht; Auftrag A05-20A; Version 1.1. Cologne. Cologne: IQWiG; 2010. https://www.iqwig.de/de/projekteergebnisse/ projekte/arzneimittelbewertung/2010-oderfrueher/a05-20a-selektiveserotonin-und-noradrenalinwiederaufnahme-hemmer-snri-zurbehandlung-derdepression.1134.html. Accessed 11 Mar 2013.

32. IQWiG. Bupropion, Mirtazapin und Reboxetin bei der Behandlung der Depression. Abschlussbericht; Auftrag A05-20C; Version 1.1., Cologne. Cologne: IQWiG; 2010. https://www.iqwig.de/de/projekte-ergebnisse/ projekte/arzneimittelbewertung/2010-oder-frueher/a05-20c-bupropionmirtazapin-und-reboxetin-zurbehandlung-der-depression.1132.html. Accessed 09 Nov 2009.

33. Glenny AM, Altman DG, Song F, Sakarovitch C, Deeks JJ, D'Amico R, Bradburn MJ, Eastwood AJ. Indirect comparisons of competing interventions. Health Technol Assess. 2005;9:1-134.

34. Wells GA, Sultan SA, Chen L, Khan M, Coyle D. Indirect Evidence: Indirect Treatment Comparisons in Meta-analysis. Ottawa: Canadian Agency for Drugs and Technologies in Health; 2009.

35. Mills EJ, Ghement I, O'Regan C, Thorlund K. Estimating the power of indirect comparisons: a simulation study. PLoS One. 2011;6:e16237.

36. Song F, Clark A, Bachmann MO, Maas J. Simulation evaluation of statistical properties of methods for indirect and mixed treatment comparisons. BMC Med Res Methodol. 2012;12:138.

37. Jonas DE, Wilkins TM, Bangdiwala S, Bann CM, Morgan LC, Thaler KJ, Amick HR, Gartlehner G. Findings of Bayesian mixed treatment comparison meta-analyses: comparison and exploration using real-world trial data and simulation. Rockville: Agency for Healthcare Research and Quality (AHRQ Publication No. 13-EHC039-EF); 2013.

38. Veroniki AA, Mavridis D, Higgins JP, Salanti G. Characteristics of a loop of evidence that affect detection and estimation of inconsistency: a simulation study. BMC Med Res Methodol. 2014;14:106.

39. Hartung J. An alternative method for meta-analysis. Biom J. 1999;41: 901-16.

40. Knapp G, Hartung J. Improved tests for a random effects meta-regression with a single covariate. Stat Med. 2003;22:2693-710.

41. Thorlund K, Thabane L, Mills EJ. Modelling heterogeneity variances in multiple treatment comparison meta-analysis - are informative priors the better solution? BMC Med Res Methodol. 2013;13:2.

42. Noma H, Tanaka S, Matsui S, Cipriani A, Furukawa TA. Quantifying indirect evidence in network meta-analysis. Stat Med. 2017;36:917-27.
43. Higgins JPT, Jackson D, Barrett JK, Lu G, Ades AE, White IR. Consistency and inconsistency in network meta-analysis: Concepts and models for multi-arm studies. Res Synth Methods. 2012;3:98-110.

44. White IR, Barrett JK, Jackson D, Higgins JPT. Consistency and inconsistency in network meta-analysis: Model estimation using multivariate meta-regression. Res Synth Meth. 2012;3:111-25.

45. Jackson D, Barrett JK, Rice S, White IR, Higgins JP. A design-by-treatment interaction model for network meta-analysis with random inconsistency effects. Stat Med. 2014;33:3639-54.

46. Jackson D, Law M, Barrett JK, Turner R, Higgins JP, Salanti G, White IR. Extending DerSimonian and Laird's methodology to perform network meta-analyses with random inconsistency effects. Stat Med. 2016;35: 819-39.

47. Law M, Jackson D, Turner R, Rhodes K, Viechtbauer W. Two new methods to fit models for network meta-analysis with random inconsistency effects. BMC Med Res Methodol. 2016;16:87.

48. Jackson D, Veroniki AA, Law M, Tricco AC, Baker R. Paule-Mandel estimators for network meta-analysis with random inconsistency effects. Res Synth Meth. 2017;8:416-34.

49. van Valkenhoef G, Kuiper J. gemtc: Network meta-analysis using Bayesian methods. R package version 0.8-2. 2016. https://CRAN.R-project.org/ package=gemtc. Accessed 03 Nov 2019.

50. Shim SR, Kim SJ, Lee J, Rücker G. Network meta-analysis: application and practice using R software. Epidemiol Health. 2019;41:e2019013.

\section{Publisher's Note}

Springer Nature remains neutral with regard to jurisdictional claims in published maps and institutional affiliations.
Ready to submit your research? Choose BMC and benefit from:

- fast, convenient online submission

- thorough peer review by experienced researchers in your field

- rapid publication on acceptance

- support for research data, including large and complex data types

- gold Open Access which fosters wider collaboration and increased citations

- maximum visibility for your research: over $100 \mathrm{M}$ website views per year

At BMC, research is always in progress.

Learn more biomedcentral.com/submissions 